\title{
Maximum Likelihood based methods for OFDM intercarrier spacing characterization
}

\author{
Abdelaziz Bouzegzi*, Philippe Ciblat ${ }^{\dagger}$, and Pierre Jallon* \\ *CEA-LETI, Grenoble, France (Email: abdelaziz.bouzegzi@cea.fr, pierre.jallon@cea.fr) \\ ${ }^{\dagger}$ ENST, Paris, France (Email: philippe.ciblat@enst.fr)
}

\begin{abstract}
One of the main task to be done by a cognitive receiver is to sense its spectral environment in order to distinguish surrounding systems from each others. Actually most systems (such as WiMAX, WiFi, DVBT) are based on OFDM modulations but differ from their intercarrier spacing used in OFDM modulation. Therefore carrying out accurate intercarrier spacing estimator is a crucial step in cognitive radio. In this paper, we propose a new efficient algorithm to estimate the intercarrier spacing based on maximum-likelihood principle. Its performance is analysed through numerical simulations and compared to standard existing approaches.
\end{abstract}

\section{INTRODUCTION}

The underlying idea for cognitive radio concept firstly introduced by [1] consists in carrying out terminal which is able to modify its transmission parameters and even its used system with respect to its own electro-magnetic environment. Therefore, first of all, a terminal based on cognitive radio concept needs to characterize its spectral environment and to recognize the standard used by others cognitive terminals/access points blindly. Most popular standards (e.g. WiFi [2], WiMAX [3], DAB [4], DVB-T [5], 3GPP/LTE [6]) are now based on OFDM modulations. However the value of their intercarrier spacing enables to distinguish them form each others. Indeed the intercarrier spacing is equal to $15.625 \mathrm{kHz}, 10.94 \mathrm{kHz}, 312.5 \mathrm{kHz}$, $1 \mathrm{kHz} 1.116 \mathrm{kHz}, 15 \mathrm{kHz}$ for Fixed WiMAX, Mobile WiMAX, WiFi, DAB, DVBT, 3GPP/LTE respectively. Consequently estimating the inter-carrier spacing of an OFDM modulated signal is equivalent to identifying the used standard. This paper thus addresses blind estimation issue of intercarrier spacing for OFDM signal. Obviously, the proposed estimation algorithm also applies to military contexts

The estimation of the intercarrier spacing for OFDM signal has already given rise to several contributions [7], [8], [9], [10], [11]. All these methods are developed in the context of cyclicprefixed OFDM and are based on the second-order statistics. Actually the useful time of the OFDM symbol (which is equal to the inverse of the intercarrier spacing) is estimated by detecting the main peak of the autocorrelation function of the received signal. Indeed, as cyclic prefix occurs, a peak occurs at the lag equal to the useful time of the OFDM symbol. When the ratio between cyclic prefix and OFDM symbol duration is small or when the multipath propagation channel is almost as large as the cyclic prefix, the autocorrelation based approach does not work well or even falls down. Obviously, under one out of these two assumptions, the peak magnitude of the autocorrelation at lag equal to the inverse of intercarrier spacing is significantly reduced.

In this paper, we propose to estimate the intercarrier spacing on relying on Maximum Likelihood principle. As no training sequence may be available, we concentrate on Non-Data Aided estimation technique. Then one can define a number of Maximum Likelihood estimators [12]. Here we focus on the so-called Deterministic Maximum Likelihood (DML) and Gaussian Maximum Likelihood (GML). Though more complex, these Maximum-Likelihood based methods are robust to small length cyclic prefix and multipath environment in opposition to autocorrelation based method. It can also be suitable for time synchronization.

This paper is organized as follows: in Section II, we describe the receive signal model thanks to matrix framework. Section III is dedicated to the introduction of the cost functions (based on the Maximum Likelihood). We also introduce simplified criteria. For sake of simplicity, novel algorithms are developed in Gaussian channel context. Section IV is devoted to numerical simulations. We especially inspect the robustness of our proposed algorithms to the presence of small cyclic prefixes, or of multipath channels, or of timing offset. Comparison with autocorrelation based method is also drawn.

\section{Signal Model}

In additive white Gaussian channel, we consider that the continuous-time OFDM receive signal takes the following form

$$
\begin{aligned}
y_{a}(t) & =\frac{1}{\sqrt{N}} \sum_{k=0}^{K-1} \sum_{n=0}^{N-1} a_{k, n} e^{-2 i \pi \frac{n\left(t-D T_{c}-k T_{s}\right)}{N T_{c}}} g_{a}\left(t-k T_{s}\right) \\
& +b_{a}(t)
\end{aligned}
$$

where $N$ is the number of subcarriers and where $1 / T_{c}$ is the information symbol rate in absence of guard interval. The intercarrier spacing is then equal to $1 / N T_{c}$. The length of the cyclic prefix is set to $D T_{c}$. The duration of a whole OFDM symbol is $T_{s}=(N+D) T_{c}$. The sequence $\left\{a_{k, n}\right\}_{k, n}$ represents the transmit unknown data symbols at subcarrier $n$ and OFDM block $k$. The shaping filter $g_{a}(t)$ is assumed to be equal to 1 if $0 \leq t<T_{s}$ and 0 otherwise. The complex-valued noise $b_{a}(t)$ is assumed to be circularly-symmetric zero-mean white Gaussian noise. Its variance is equal to $N_{0}$ per real dimension. Finally we consider the transmission of $K$ OFDM symbols for 
an observation window of duration $T_{0}$. We have $K=\left\lceil T_{0} / T_{s}\right\rceil$ where $\lceil X\rceil$ stands for the smallest integer not less than $X$.

The continuous-time received signal $y_{a}(t)$ is sampled at sampling frequency $1 / T_{e}$ where $T_{e}$ is the sampling period. The ratio $q=T_{c} / T_{e}$ denotes the sampling factor. The discretetime receive signal is denoted by $y(m)=y_{a}\left(m T_{e}\right)$. In order to keep the information carrying by the continuous-time signal, the sampling frequency must be larger than the OFDM signal bandwidth, i.e., greater than $1 / T_{c}$. The number of available samples is then equal to $M=\left\lfloor T_{0} / T_{e}\right\rfloor$ where $\lfloor X\rfloor$ stands for the largest integer not greater than $X$. We get

$$
\begin{aligned}
y(m) & =\frac{1}{\sqrt{N}} \sum_{k=0}^{K-1} \sum_{n=0}^{N-1} a_{k, n} e^{-2 i \pi n m \frac{T_{e}}{N T_{c}}} e^{2 i \pi n(k+1) \frac{D T_{c}}{N T_{c}}} \\
& \times g_{a}\left(m T_{e}-k(N+D) T_{c}\right)+b(m)
\end{aligned}
$$

with $b(m)=b_{a}\left(m T_{e}\right)$.

In practice, the terminal just has the knowledge of $\{y(m)\}_{m=0}^{M-1}, M, T_{0}, T_{e}$ and wishes to estimate the values of $N, N T_{c}, D T_{c}$. For selecting the used standard, the cognitive terminal firstly needs the knowledge of the intercarrier spacing, given by the inverse of $N T_{c}$. Notice that, in Eq. (2), $K$ and $a_{k, n}$ are unknown as well.

We stack all the receive samples in an unique vector $\mathbf{y}=$ $[y(0), \cdots, y(M-1)]^{\mathrm{T}}$ where $(.)^{\mathrm{T}}$ stands for the transposition. Since Eq. (2) is linear with respect to transmit data, it exists a matrix $\mathbf{H}_{\boldsymbol{\theta}}$ of size $M \times K N$ depending on $N, D T_{c}$ et $N T_{c}$ such that

$$
\mathbf{y}=\mathbf{H}_{\boldsymbol{\theta}} \mathbf{a}+\mathbf{b}
$$

where

$$
\begin{aligned}
& \text { - } \boldsymbol{\theta}=\left[N, D T_{c}, N T_{c}\right] \\
& \text { - } \mathbf{a}_{k}=\left[a_{k, 0}, \cdots, a_{k, N-1}\right]^{\mathrm{T}} \text { of size } N \times 1 \\
& \text { - } \mathbf{a}=\left[\mathbf{a}_{0}^{\mathrm{T}}, \cdots, \mathbf{a}_{K-1}^{\mathrm{T}}\right]^{\mathrm{T}} \text { of size } K N \times 1 \\
& \text { - } \mathbf{b}=[b(0), \cdots, b(M-1)]^{\mathrm{T}} \text { of size } M \times 1
\end{aligned}
$$

Before going further, we will obtain a closed-form expression for $\mathbf{H}_{\boldsymbol{\theta}}$. As $g_{a}(t)$ is only non-null on the interval $\left[0, T_{s}\right]$, we have

$$
0 \leq m T_{e}-k(N+D) T_{c}<(N+D) T_{c}
$$

which implies that

$$
m \frac{T_{e}}{(N+D) T_{c}}-1<k \leq m \frac{T_{e}}{(N+D) T_{c}} .
$$

Consequently, for a given $m$, it exists only an unique value of $k$, denoted by $k_{m} . \mathbf{H}_{\boldsymbol{\theta}}$ is then composed by null components except the next ones

$$
H_{\boldsymbol{\theta}}\left(m, k_{m} N+n\right)=\frac{1}{\sqrt{N}} e^{-2 i \pi n m \frac{T_{e}}{N T_{c}}} e^{2 i \pi n\left(k_{m}+1\right) \frac{D T_{c}}{N T_{c}}}
$$

for $m=0, \ldots, M-1$ and $n=0, \cdots, N-1$.

Once this signal model provided, we are able to carry out Maximum-Likelihood based estimators for parameters $N$, $D T_{c}$, and $N T_{c}$. We remind that we are particularly interested by the estimation of $N T_{c}$.

\section{MAXIMUM-LIKELIHOOD BASED ALGORITHMS}

In blind estimation context, we recall that transmit data a introduced in Eq. (3) are unknown at the receiver. Consequently carrying out the true Maximum-Likelihood based estimator of $N, D T_{c}$, and $N T_{c}$ is complex since the likelihood of $\mathbf{y}$ given a, $N, D T_{c}$, and $N T_{c}$ has to be averaged over the vector a. To overcome the problem, it is proposed to consider the vector a as parameters of interest too which leads to the so-called Deterministic Maximum Likelihood or to consider vector a as Gaussian (even if a is not Gaussian vector) which leads to the so-called Gaussian Maximum-Likelihood [12].

\section{A. Deterministic Maximum-Likelihood approach (DML)}

Let $p(\mathbf{y} \mid \tilde{\boldsymbol{\theta}}, \tilde{\mathbf{a}})$ be the likelihood of $\mathbf{y}$ given the trial values $\tilde{\boldsymbol{\theta}}=\left[\tilde{N}, \widetilde{D T_{c}}, \widetilde{N T}_{c}\right]$ and $\tilde{\mathbf{a}}$. The deterministic MaximumLikelihood is defined as follows [12]

$$
[\hat{\boldsymbol{\theta}}, \hat{\mathbf{a}}]=\arg \max _{\tilde{\boldsymbol{\theta}}, \tilde{\mathbf{a}}} p(\mathbf{y} \mid \tilde{\boldsymbol{\theta}}, \tilde{\mathbf{a}})
$$

In practice, the signal bandwidth (given by $1 / T_{c}$ ) can be assumed to be roughly known. This enables us to choose a reasonnable value for $1 / T_{e}$ and also to filter the receive signal by an ideal low-pass filter of unit-magnitude and bandwidth $1 / T_{c}$. This induces that the discrete-time noise has the following autocorrelation function

$$
r_{b}(n)=\mathbb{E}[b(m+n) \overline{b(m)}]=\frac{2 N_{0}}{T_{c}} \operatorname{sinc}\left(\frac{\pi n}{q}\right) .
$$

The discrete-time noise is not white. In order to simplify the DML estimator, the discrete-time noise will be however assumed to be white. Obviously, in simulation part, the noise process color will not be neglected.

By assuming the noise vector $\mathbf{b}$ uncorrelated and by considering $K N \leq M$, it is well known that the DML estimator can take the following form [12]

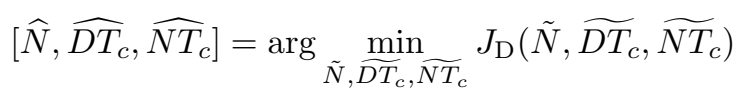

with

$$
J_{\mathrm{D}}\left(\tilde{N}, \widetilde{D T_{c}}, \widetilde{N T_{c}}\right)=\left\|\left(\mathbf{I d}_{M}-\mathbf{H}_{\tilde{\boldsymbol{\theta}}}\left(\mathbf{H}_{\tilde{\boldsymbol{\theta}}}^{\mathrm{H}} \mathbf{H}_{\tilde{\boldsymbol{\theta}}}\right)^{-1} \mathbf{H}_{\tilde{\boldsymbol{\theta}}}^{\mathrm{H}}\right) \mathbf{y}\right\|
$$

and where $(.)^{\mathrm{H}}$ stands for the conjugate-transposition.

If $T_{0} / T_{s}$ is an integer, one can prove that

$$
\mathbf{H}_{\tilde{\boldsymbol{\theta}}}^{\mathrm{H}} \mathbf{H}_{\tilde{\boldsymbol{\theta}}}=\tilde{q}\left(1+\frac{\widetilde{D T_{c}}}{\widetilde{N T_{c}}}\right) \mathbf{I d}_{\tilde{K} \tilde{N}} .
$$

If $T_{0} / T_{s}$ is not an integer, the previous equation does not hold. Nevertheless, in order to remove the matrix inversion in Eq. (4) and to simplify the DML estimator, we propose an approximate DML estimator where $\mathbf{H}_{\tilde{\boldsymbol{\theta}}}^{\mathrm{H}} \mathbf{H}_{\tilde{\boldsymbol{\theta}}}$ is replaced with $\tilde{q}\left(1+\widetilde{D T}_{c} / \widetilde{N T}_{c}\right) \mathbf{I d}_{\tilde{K} \tilde{N}}$ even if $T_{0} / T_{s}$ is not an integer. Thus we obtain

$$
\left[\widehat{N}, \widehat{D T}_{c}, \widehat{N T}_{c}\right]=\arg \min _{\tilde{N}, \widetilde{D T}_{c}, \widetilde{N T}_{c}} J_{\mathrm{AD}}\left(\tilde{N}, \widetilde{D T}_{c}, \widetilde{N T}_{c}\right)
$$


with

$$
J_{\mathrm{AD}}\left(\tilde{N}, \widetilde{D T_{c}}, \widetilde{N T_{c}}\right)=\left\|\left(\mathbf{I d}_{M}-\frac{\mathbf{H}_{\tilde{\boldsymbol{\theta}}} \mathbf{H}_{\tilde{\boldsymbol{\theta}}}^{\mathrm{H}}}{\tilde{q}\left(1+\widetilde{D T_{c}} / \widetilde{N T_{c}}\right)}\right) \mathbf{y}\right\|
$$

Notice that $\tilde{q}$ depends on the trial value $\tilde{\boldsymbol{\theta}}$.

\section{B. Gaussian Maximum-Likelihood approach (GML)}

In this subsection, the transmit data vector $\mathbf{a}$ is assumed to be an i.i.d. random vector. Its true power density function (pdf) is a product of a sum of Dirac distribution for which the location is given by the used constellation (either PAM or PSK or QAM). Due to the high complexity of derivations, it is usual to model the vector a as a circularly-symmetric Gaussian multivariate process with zero mean and covariance $\sigma_{a}^{2}$ per real dimension [12]. Then the so-called Gaussian likelihood, denoted by $p_{g}(\mathbf{y} \mid \tilde{\boldsymbol{\theta}})$, can be expressed in closed-form when a is assumed as above.

Consequently the multivariate process $\mathbf{y}$ is circularlysymmetric Gaussian process with zero mean and covariance matrix $\mathbb{E}\left[\mathbf{y y}^{\mathrm{H}}\right]=2 \sigma_{a}^{2} \mathbf{H}_{\boldsymbol{\theta}} \mathbf{H}_{\boldsymbol{\theta}}^{\mathrm{H}}+2 N_{0} \mathbf{I} \mathbf{d}_{M}$ and yields the following likelihood

$$
\begin{aligned}
p_{g}(\mathbf{y} \mid \tilde{\boldsymbol{\theta}}) & =\frac{1}{(2 \pi)^{M} \operatorname{det}\left(2 \sigma_{a}^{2} \mathbf{H}_{\tilde{\boldsymbol{\theta}}} \mathbf{H}_{\tilde{\boldsymbol{\theta}}}^{\mathrm{H}}+2 N_{0} \mathbf{I} \mathbf{d}_{M}\right)} \\
& \times e^{-\frac{1}{2} \mathbf{y}^{\mathrm{H}}\left(\sigma_{a}^{2} \mathbf{H}_{\tilde{\boldsymbol{\theta}}} \mathbf{H}_{\tilde{\boldsymbol{\theta}}}^{\mathrm{H}}+N_{0} \mathbf{I d}_{M}\right)^{-1} \mathbf{y}}
\end{aligned}
$$

Let $\mathbf{I d}$ and $\mathbf{A}$ be the identity matrix and an other matrix compatible in size respectively. We remind that $\operatorname{det}(\mathbf{I d}+$ $\left.\mathbf{A} \mathbf{A}^{\mathrm{H}}\right)=\operatorname{det}\left(\mathbf{I d}+\mathbf{A}^{\mathrm{H}} \mathbf{A}\right)$ and $\left(\mathbf{I d}+\mathbf{A} \mathbf{A}^{\mathrm{H}}\right)^{-1}=\mathbf{I d}-\mathbf{A}(\mathbf{I d}+$ $\left.\mathbf{A}^{\mathrm{H}} \mathbf{A}\right)^{-1} \mathbf{A}^{\mathrm{H}}$. This leads to

$$
\begin{aligned}
p_{g}(\mathbf{y} \mid \tilde{\boldsymbol{\theta}}) & \propto \frac{1}{\operatorname{det}\left(2 \sigma_{a}^{2} \mathbf{H}_{\tilde{\boldsymbol{\theta}}}^{\mathrm{H}} \mathbf{H}_{\tilde{\boldsymbol{\theta}}}+2 N_{0} \mathbf{I} \mathbf{d}_{\tilde{K} \tilde{N}}\right)} \\
& \times e^{\frac{\sigma_{a}^{2}}{N_{0}} \mathbf{y}^{\mathrm{H}} \mathbf{H}_{\tilde{\boldsymbol{\theta}}}\left(2 \sigma_{a}^{2} \mathbf{H}_{\tilde{\boldsymbol{\theta}}}^{\mathrm{H}} \mathbf{H}_{\tilde{\boldsymbol{\theta}}}+2 N_{0} \mathbf{I d}_{\tilde{K} \tilde{N}}\right)^{-1} \mathbf{H}_{\tilde{\boldsymbol{\theta}}}^{\mathrm{H}} \mathbf{y}}
\end{aligned}
$$

As maximizing $p_{g}(\mathbf{y} \mid \tilde{\boldsymbol{\theta}})$ is equivalent to minimizing $-\log p_{g}(\mathbf{y} \mid \tilde{\boldsymbol{\theta}})$, we get

$$
\left[\widehat{N}, \widehat{D T}_{c}, \widehat{N T}_{c}\right]=\arg \underset{\tilde{N}, \min _{\widetilde{D T}}, \widetilde{N T_{c}}}{ } J_{\mathrm{G}}\left(\tilde{N}, \widetilde{D T_{c}}, \widetilde{N T_{c}}\right)
$$

with

$$
\begin{aligned}
J_{\mathrm{G}}\left(\tilde{N}, \widetilde{D T_{c}}, \widetilde{N T_{c}}\right) & =\log \left(\operatorname{det}\left(2 \sigma_{a}^{2} \mathbf{H}_{\tilde{\boldsymbol{\theta}}}^{\mathrm{H}} \mathbf{H}_{\tilde{\boldsymbol{\theta}}}+2 N_{0} \mathbf{I d}_{\tilde{K} \tilde{N}}\right)\right) \\
& -\frac{\sigma_{a}^{2}}{2 N_{0}} \mathbf{y}^{\mathrm{H}} \mathbf{H}_{\tilde{\boldsymbol{\theta}}}\left(\sigma_{a}^{2} \mathbf{H}_{\tilde{\boldsymbol{\theta}}}^{\mathrm{H}} \mathbf{H}_{\tilde{\boldsymbol{\theta}}}+N_{0} \mathbf{I} \mathbf{d}_{\tilde{K} \tilde{N}}\right)^{-1} \mathbf{H}_{\tilde{\boldsymbol{\theta}}}^{\mathrm{H}} \mathbf{y} .
\end{aligned}
$$

Notice that $\tilde{K}$ depends on the trial value $\tilde{\boldsymbol{\theta}}$. Similar estimator has been already introduced by [13] in the context of symbol period estimation for single carrier modulated signal.

Once again, if Eq. (5) holds, the GML estimator can be well approximated by

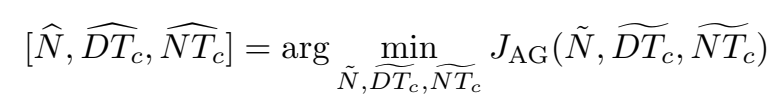

with

$$
\begin{aligned}
J_{\mathrm{AG}}\left(\tilde{N}, \widetilde{D T_{c}}, \widetilde{N T_{c}}\right) & =\tilde{K} \tilde{N} \ln \left(2 \sigma_{a}^{2} \tilde{q}\left(1+\frac{\widetilde{D T_{c}}}{\widetilde{N T}_{c}}\right)+2 N_{0}\right) \\
& -\frac{\left(\sigma_{a}^{2} / N_{0}\right)}{2\left(\sigma_{a}^{2} \tilde{q}\left(1+\frac{\widetilde{D T_{c}}}{\widetilde{N T_{c}}}\right)+N_{0}\right)} \mathbf{y}^{\mathrm{H}} \mathbf{H}_{\tilde{\boldsymbol{\theta}}} \mathbf{H}_{\tilde{\boldsymbol{\theta}}}^{\mathrm{H}} \mathbf{y} .
\end{aligned}
$$

Notice that $\tilde{q}$ and $\tilde{K}$ depend on the tested parameters $\tilde{\boldsymbol{\theta}}$. So, as done in [13], the first term of the RHS can not be removed. Moreover Signal-to-Noise Ratio (provided by $\sigma_{a}^{2} / N_{0}$ ) has to be estimated prior to computing GML estimators.

\section{Simulations}

This section is devoted to numerical simulations in order to evaluate the performance and the merit of proposed algorithms. Except otherwise stated, the number of carriers $(N)$ is fixed to 64 , the ratio between the cyclic prefix length over the useful OFDM symbol time $(\mathrm{CP}:=D / N)$ is equal to $1 / 8$. The duration of the useful part of OFDM symbol $\left(N T_{c}\right)$ is $64 \mu \mathrm{s}$. The number of available OFDM symbols $\left(T_{0} / T_{s}\right)$ is 5 . The sampling rate $T_{e}$ is chosen as $T_{e}=T_{c} / 2$, i.e., $q=2$.

In practice, the receive continuous-time signal is not only disturbed by additive white Gaussian noise but also by multipath propagation continuous-time channel for which the dispersion time is denoted by $T_{d}$. Actually the receive discretetime signal, now denoted by $\tilde{y}(m)$, can be modeled as

$$
\tilde{y}(m)=\sum_{\ell=0}^{L} h_{\ell} x(m-\ell)+b(m)
$$

where $x(m)$ is the noiseless part of $y(m)$ defined by Eq. (2) and where $L=\left\lceil T_{d} / T_{e}\right\rceil$. Each component of channel impulse response is assumed to be Gaussian distributed with zero mean and same variance. Then each channel realization is normalized. Unless otherwise stated, the continuous-time channel dispersion time is fixed to be a quarter of the cyclic prefix duration, which means that $T_{d} / D T_{c}=1 / 4$. No frequency offset has been considered. We firstly assume that the terminal is perfectly time-synchronized. Each curve is averaged over 1000 Monte-Carlo runs. The SNR is defined as $\sigma_{a}^{2} / N_{0}$ and fixed to $10 \mathrm{~dB}$.

As our practical issue related to cognitive radio consists in operating system identification, we are only interested to the estimation accuracy of $N T_{c}$. Therefore, in the sequel, the performance on $N$ and $D T_{c}$ is omitted. Actually we have observed that performance on $N T_{c}, D T_{c}$, or $N$ always yields close performance. In addition, identifying the right system (WiMAX, WiFi, DAB, DVB-T or 3GPP/LTE, etc) boils down to comparing $\widehat{N T}_{c}$ to its theoretical value for each considered system. Since the smallest gap between two inter-carrier spacing values is little larger than $1 \%$ (cf. Section I), we do not need a tight estimation of $N T_{c}$ but we only need an estimation of $1 / N T_{c}$ up to $1 \%$. Consequently rather than considering the Mean Square Error as usually done in estimation issue, we prefer considering the detection rate defined as follows: we assert that the inter-carrier spacing estimation is correct if $1 / \widehat{N T}_{c}$ is close to $1 / N T_{c}$ up to $1 \%$. 
In practice, to obtain $\widehat{N T}_{c}$, we have calculated the cost function for each trial $\widetilde{N T}$ c belonging to the grid of step $0.64 \mu \mathrm{s}$ starting at $25 \mu \mathrm{s}$ and ending at $100 \mu \mathrm{s}$. This leads to a gap equal to $1 \%$ between two adjacent tested intercarrier spacing compared to the true value $64 \mu \mathrm{s}$. For each considered value of $\widetilde{N T}_{c}, \widetilde{D T}_{c}$ takes values in the set $\widetilde{N T}_{c} \times$ $\{1 / 2,1 / 4,1 / 8,1 / 16,1 / 32\}$, and $\widetilde{N}$ takes its value in the set $\{256,128,64,32\}$. Finally the performance has been evaluated as the number of wrong detection, i.e. the number of realizations for which the $\widehat{N T}_{c}$ is different from $N T_{c}$ up to $0.64 \mu \mathrm{s}$.

In Figure 1, we display the wrong detection rate for proposed algorithms and autocorrelation based method (denoted by $\mathrm{COR}$ ) versus $\mathrm{CP}$ in AWGN context (i.e., $T_{d}=0$ ). We remark that if $\mathrm{CP}$ is equal to $1 / 4$, proposed algorithms and autocorrelation based algorithm offer the same performance. In contrast, our algorithms are more robust to small values of CP. For instance AGML and ADML algorithm still work well in absence of cyclic prefix. Notice that standard CP varies from 1/32 (DVB-T in France) to $1 / 4$ (WiFi). Consequently, our proposed algorithms are more appropriate for the cognitive radio than the autocorrelation based method. Indeed, autocorrelation method does not enable system identification if encountered systems employ small cyclic prefix as done in DVB-T and some Wimax configurations.

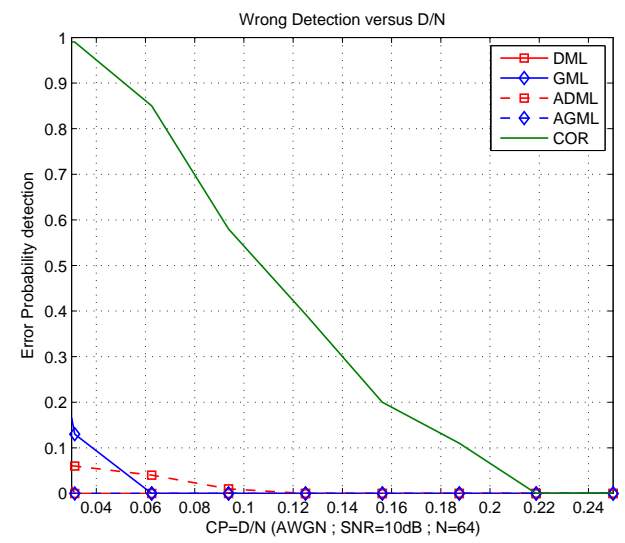

Fig. 1. Wrong detection rate vs. CP

In Figure 2, we plot the wrong detection rate versus $T_{d} / D T_{c}$ for proposed algorithms and autocorrelation based method. We oberve that our algorithms are more robust to the presence of multipath channels. For instance, even if the channel impulse response lies on the entire cyclic prefix, DML, GML and AGML still perform well. In contrast, the autocorrelation based method falls down as soon as the continuous-time channel length is more than $D T_{c} / 10$. Notice that the performance of the autocorrelation based method in previous-cited papers is usually evaluated by numerical simulations with $\mathrm{CP}=1 / 4$ and AWGN which prevents to show these drawbacks.

In Figure 3, we plot the wrong detection rate versus SNR for proposed algorithms and autocorrelation based method. As $\mathrm{CP}=1 / 8$ and $T_{d} / D T_{c}=1 / 4$, the autocorrelation based

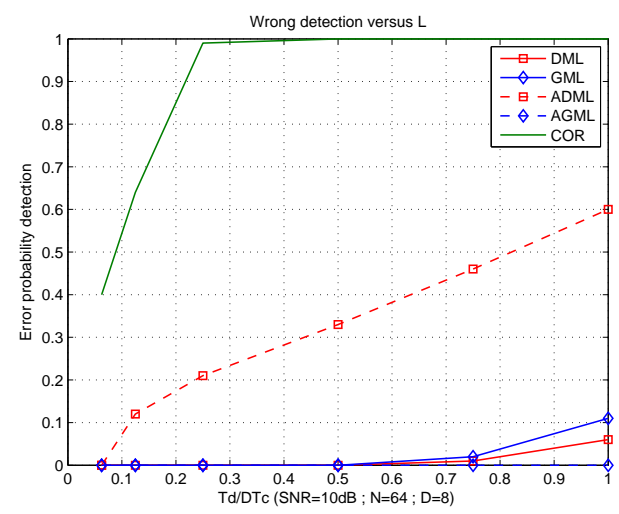

Fig. 2. Wrong detection rate vs. $T_{d} / D T_{c}$

method fails whatever the SNR. Proposed algorithms (especially, GML, and AGML) are able to offer good performace even at low SNR such as OdB.

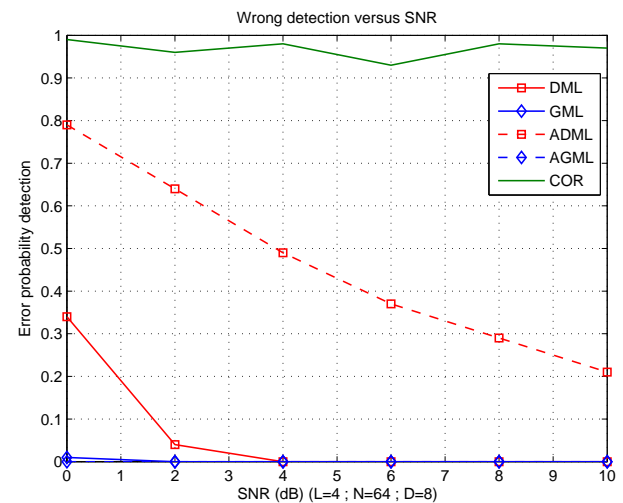

Fig. 3. Wrong detection rate vs. SNR

In Figure 4, we analyse the wrong detection rate versus $T_{0} / T_{s}$ for proposed algorithms and autocorrelation based method. Once again, as $\mathrm{CP}=1 / 8$ and $T_{d} / D T_{c}=1 / 4$, the autocorrelation based method fails whatever the observation window length. In contrast, proposed algorithms (especially, GML, and AGML) yield interesting performance even when one OFDM symbol is available. We remark that in four already-introduced figures, the Gaussian ML algorithms are better than the Deteministic ones. Morever, the AGML is better than GML and easier to be computed since it does not require matrix inversion.

We now consider that the receive signal is not timesynchronized that is to say that the beginning of the receive signal does not coincide with the beginning of an OFDM symbol. Then the receive signal $\tilde{y}(m)$ takes the following form

$$
\tilde{y}(m)=\sum_{\ell=0}^{L} h_{\ell} x(m-\ell-\tau)+b(m) .
$$

In Figure 5, we inspect the wrong detection rate versus $\tau / T_{s}$ 


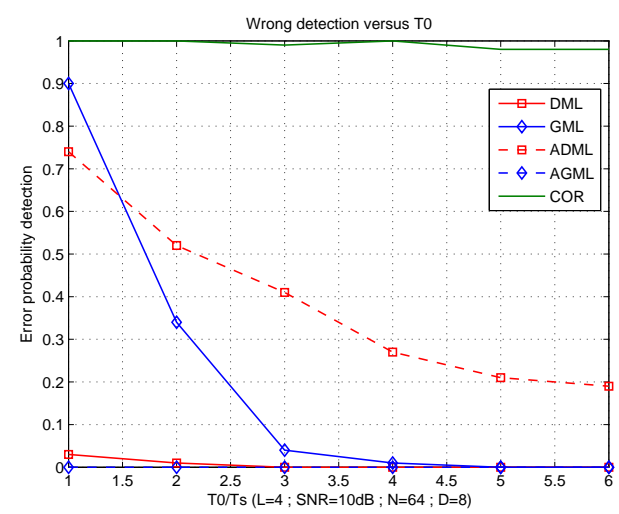

Fig. 4. Wrong detection rate vs. $T_{0} / T_{s}$

for proposed algorithms and autocorrelation based method in AWGN context $\left(T_{d}=0\right)$. Our algorithms are just slightly robust to time offset. As it is well-known, the autocorrelation is insensitive to time offset. Consequently to carrying out proposed algorithms, we need either implementing a prior time-synchronisation or adding parameter $\tau$ to the parameters of interest $\boldsymbol{\theta}$.

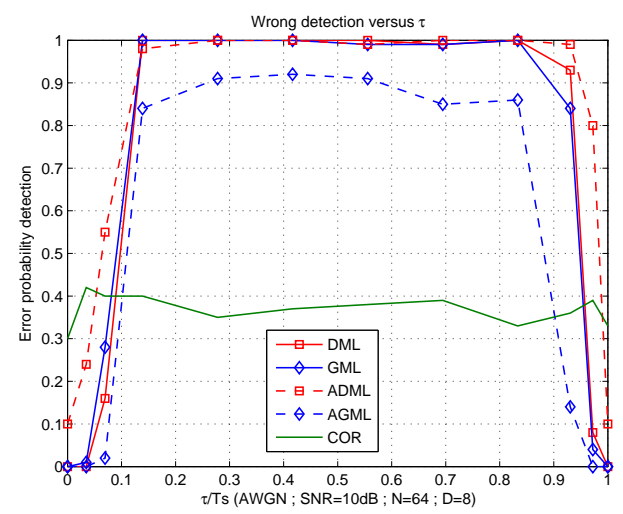

Fig. 5. Wrong detection rate vs. $\tau / T_{s}$

In Figure 6, we modify our algorithms by considering the vector of parameters $\boldsymbol{\theta}=\left[N, D T_{c}, N T_{c}, \tau\right]$. We plot the performance of proposed algorithms and autocorrelation based method versus SNR when the signal is time-desynchronized and when the channel is AWGN. Compared to Figure 3 (done in frequency-selective channel context and not in AWGN context), we observe that our algorihms are now insensitive to missynchronisation. In return, we have increased the complexity of our algorithms.

We now consider that the received signal is not perfectly in baseband. Consequently the receive signal writes as

$$
\tilde{y}(m)=e^{2 i \pi \Delta f m} \sum_{\ell=0}^{L} h_{\ell} x(m-\ell)+b(m)
$$

where $\Delta f$ is the so-called frequency offset. Due to the lack of space, we do not display the associated figures. Actually

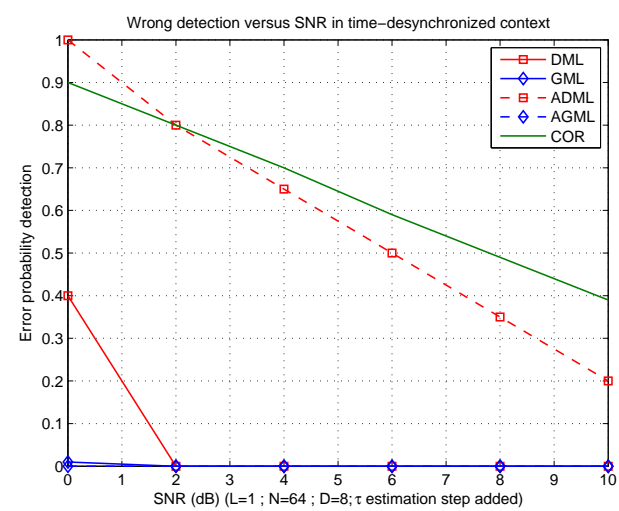

Fig. 6. Wrong detection rate vs. SNR in time-missynchronisation context

we have observed that our algorithms are once again very sensitive to frequency offset whereas autocorrelation based method performance is independent of frequency offset. As done in time-missynchronisation context, the frequency offset has to be added into the vector of parameters of interest.

\section{CONCLUSION}

In this paper, we proposed new ML based algorithms. Their performance has been evaluated by means of numerical simulations. We showed that new methods outperform autocorrelation based method in some useful contexts.

\section{REFERENCES}

[1] J. Mitola, Cognitive Radio : an Integrated Agent architecture for Software Defined Radio, Phd thesis, Royal Institute of Technology (Stockholm, Sweden), 2000.

[2] C. Smith and J. Meyer, $3 G$ wireless with Wimax and $\mathrm{Wi}-\mathrm{Fi}: 802.16$ and 802.11, McGraw-Hill, 2005.

[3] L. Nuaymi, Wimax: technology for broadband wireless access, John Wiley, 2007.

[4] W. Hoeg and T. Lauterbach, Digital Audio Broadcasting: principles and applications of digital radio, John Wiley, 2003.

[5] ETSI, "Digital videl broadcasting (DVB) framing structure, channel coding and modulation for digital terrestrial television," in ETSI Report, November 2004.

[6] H. Holma and A. Toskala, WCDMA for UMTS: HSDPA evolution and LTE, John Wiley, 2007.

[7] P. Liu, B.-B. Li, Z.-Y. Lu, and F.-K. Gong, "A blind time-parameters estimation scheme for OFDM in multi-path channel," in International Conference on Wireless Communications, Networking and Mobile Computing, Sept. 2005, pp. 242-247.

[8] B. Wang and L. Ge, "Blind identification of OFDM signal in rayleigh channels," in International Conference on Wireless Communications, Networking and Mobile Computing, Dec. 2005, pp. 950-954.

[9] H. Ishii and G.W. Wornell, "OFDM blind parameter identification in cognitive radios," in IEEE International Conference on Personal, Indoor and Mobile Radio Communications, Sept. 2005, pp. 700-705.

[10] D. Landstrom, S.K. Wilson, J-J Van de Beek, P. Odling, and P.O. Borjesson, "Symbol time offset estimation in coherent OFDM systems," in IEEE Trans. on Communications, April. 2002.

[11] W. Akmouche, E. Kerhervé, and A. Quinquis, "Estimation of OFDM signal parameters : Time parameters," in Asilomar Conference, Nov. 2000.

[12] G. Vazquez and J. Riba, "Non data-aided digital synchronization," Edition on Signal Processing Advances in Communications, 2000.

[13] C. Mosquera, S. Scalise, and R. Lopez-Valcarce, "Symbol rate estimation for DVB-S2 broadcasting," in International Workshop on Signal Processing for Space Communications, 2006. 